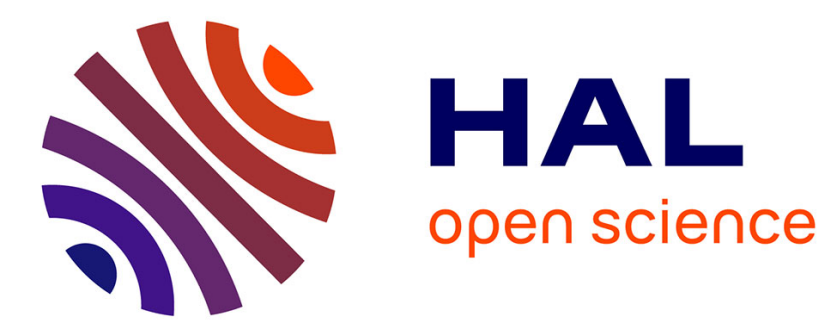

\title{
The Development of Group Stereotypes from Descriptions of Group Members: An Individual Difference Approach
}

Kimberley A. Clow, Victoria M. Esses

\section{- To cite this version:}

Kimberley A. Clow, Victoria M. Esses. The Development of Group Stereotypes from Descriptions of Group Members: An Individual Difference Approach. Group Processes and Intergroup Relations, 2005, 8 (4), pp.429-445. 10.1177/1368430205056469 . hal-00571616

\section{HAL Id: hal-00571616 https://hal.science/hal-00571616}

Submitted on 1 Mar 2011

HAL is a multi-disciplinary open access archive for the deposit and dissemination of scientific research documents, whether they are published or not. The documents may come from teaching and research institutions in France or abroad, or from public or private research centers.
L'archive ouverte pluridisciplinaire HAL, est destinée au dépôt et à la diffusion de documents scientifiques de niveau recherche, publiés ou non, émanant des établissements d'enseignement et de recherche français ou étrangers, des laboratoires publics ou privés. 


\title{
The Development of Group Stereotypes from Descriptions of Group Members: An Individual Difference Approach
}

\author{
Kimberley A. Clow and Victoria M. Esses \\ University of Western Ontario
}

\begin{abstract}
This research examined the effects of Personal Need for Structure, Need for Closure, and Personal Fear of Invalidity on information processing during the development of stereotypes. In Study 1, participants read as many group member descriptions as they wanted before expressing group stereotypes. Participants higher in Personal Fear of Invalidity sought more information; they also developed more detailed stereotypes when they received more information, whereas participants lower in Personal Fear of Invalidity did not. There was a tendency for participants higher in Need for Structure \& Closure to develop less accurate stereotypes. Finally, participants higher in Need for Structure \& Closure or Personal Fear of Invalidity were less confident about their stereotypes when they received more information, whereas participants lower in Need for Structure \& Closure or Personal Fear of Invalidity were more confident. In Study 2, participants were presented with two, four, or eight descriptions of group members before expressing stereotypes. Participants lower in Personal Fear of Invalidity developed more detailed stereotypes when they received more information, whereas participants higher in Personal Fear of Invalidity did not. When two or eight group member descriptions were presented (fewer or more than participants probably would have chosen themselves), participants higher in Personal Fear of Invalidity and lower in Need for Structure \& Closure generated the most accurate stereotypes. Finally, participants higher in Need for Structure \& Closure did not differ in stereotype confidence as a function of how much information they received, whereas participants lower in Need for Structure \& Closure were more confident when they received more information. These results indicate that cognitive style plays a role in the development of group stereotypes.
\end{abstract}

KEYWORD S Need for Closure, Personal Fear of Invalidity, Personal Need for Structure, stereotype development, stereotypes

Stereotypes have been defined as beliefs about the specific characteristics possessed by members of a social group (Esses, Haddock, \& Zanna, 1993). Utilizing stereotypes-relying on general beliefs-is one way to reduce the expenditure of cognitive resources (Macrae,

\section{Author's note}

Address correspondence to Kimberley A. Clow, Faculty of Social Science, University of Ontario Institute of Technology, 2000 Simcoe Street North, Oshawa, Ontario, Canada L1H 7K4 [email: kimberley.clow@uoit.ca] 
Milne, \& Bodenhausen, 1994). When interacting with a new group member, for example, one can reduce the amount of information that must be gathered about that person if one can use information previously gathered about other group members. In general, people prefer to process information in a relatively effortless and efficient manner, and so they often stereotype people and groups (e.g. Chaiken, Liberman, \& Eagly, 1989; Fiske \& Neuberg, 1990).

Because stereotyping is common, several researchers have investigated the development of stereotypes and the factors that may influence that development. For example, such factors as trait variability (e.g. Dijksterhuis \& van Knippenberg, 1999), group variability (e.g. Ryan, Bogart, \& Vender, 2000), in-group versus out-group membership (e.g. Ryan \& Bogart, 1997), group entitativity (e.g. McConnell, Sherman, \& Hamilton, 1997) and crossedcategorization (e.g. Meiser \& Hewstone, 2001) have all been found to influence the development of group stereotypes.

Researchers have also suggested that information processing styles may play a role (e.g. Hamilton \& Trolier, 1986; Sherman, 2001; Stroessner \& Plaks, 2001). Three important aspects of information processing that are relevant to stereotype development are: (1) the amount of information people seek on which to base their stereotypes, (2) the content of the stereotypes that are developed, and (3) the confidence people have in the stereotypes that they develop. The amount of information on which a stereotype is based dictates the possible content of the stereotype, with more information allowing for greater variability in content. The content of the stereotype that is developed, such as its detail and accuracy, can affect when and how the stereotype is utilized and how amenable it is to change. Finally, stereotype confidence is likely to affect how often the stereotype is used and whether it is altered. When confidence is high, stereotypes are used more often and are less likely to be altered.

Past research on information processing has demonstrated that information seeking, accuracy, and confidence can be shaped by individual differences (see Chaiken et al., 1989; Moskowitz, 1993; Neuberg \& Newsom, 1993; Schaller, Boyd, Yohannes, \& O'Brien, 1995; Webster \& Kruglanski, 1994). Our research investigates the relations among three individual differences-Personal Need for Structure, Need for Closure, and Personal Fear of Invalidityand their role in stereotype development.

\section{Individual differences}

\section{Relations among the constructs}

Personal Need for Structure, Need for Closure, and Personal Fear of Invalidity were all derived from Kruglanski's (1989) theory of lay epistemology, which was developed to explain how people obtain knowledge. In his theory, Kruglanski proposed two opposing motivations that drive information processing - the fear of making incorrect decisions and the need to keep life simple. From this theory, Thompson, Naccarato, and Parker (1989) derived the Personal Fear of Invalidity Scale, which measures the fear of making incorrect decisions, and the Personal Need for Structure Scale, which measures the need to lead a simple, structured life. Later, Webster and Kruglanski (1994) created the Need for Closure Scale, which measures the need for answers and a fear of ambiguity and confusion.

Conceptually, the constructs measured by these scales are very similar. Fear of committing to answers because they might be wrong (Personal Fear of Invalidity) should be negatively related to the need to seek answers to questions and avoid ambiguity and confusion (Need for Closure), and the desire for a simple, structured lifestyle (Personal Need for Structure). Moreover, the need for a simple, structured lifestyle should be positively related to the need to seek answers and avoid ambiguity and confusion.

Although there is widespread agreement that these three constructs are similar, researchers disagree about the degree to which they are related. Webster and Kruglanski (1994) found that the correlation between Need for Closure and Personal Need for Structure was weak $(r=$ $.24)$, whereas Neuberg, Judice, and West (1997) 
and Neuberg, West, Judice, and Thompson (1997) found that it was strong $(r=.79)$. More recently, Kruglanski et al. (1997) agreed that Personal Need for Structure and the Need for Closure are correlated more strongly than they first thought (correlations of .67 to .79), but noted that 'this leaves about $50 \%$ of the variance in the two scales unexplained by their commonality' (p. 1007). The correlation between scores on these two scales, however, is about the same as the reliabilities of the scales (.77 to .84; Neuberg \& Newsom, 1993; Webster \& Kruglanski, 1994), suggesting that both scales may be assessing the same thing. In contrast, there is no evidence that Personal Fear of Invalidity correlates significantly with either Personal Need for Structure or Need for Closure.

\section{Information processing}

Research on Personal Need for Structure has shown that when this need is strong, people are more likely to generate spontaneous trait inferences (Moskowitz, 1993), develop less accurate stereotypes in an illusory correlation paradigm (Schaller et al., 1995), and stereotype others (Neuberg \& Newsom, 1993). They are also less likely to change their personal views about others in light of new information (Neuberg \& Newsom, 1993). Research on Need for Closure has shown that when this need is strong, people seek only minimal information prior to forming impressions (Webster \& Kruglanski, 1994, Study 4), form less accurate impressions (Webster \& Kruglanski, 1994, Study 5), and are more confident in the impressions that they form (Webster \& Kruglanski, 1994, Study 4 and Study 5).

Thus, past research on the role of Personal Need for Structure and Need for Closure in impression formation and stereotype usage shows that these individual differences are related to differences in information processing. Although no one has investigated Personal Fear of Invalidity in this regard, its relations to Personal Need for Structure and Need for Closure suggest that Personal Fear of Invalidity may be important as well. To the best of our knowledge, we are the first to examine the role of all three constructs in stereotype development. Our research is also unusual because we allowed participants to choose the amount of information they received in one study, and then manipulated the amount of information that participants received in a second study.

\section{Operationalizing information}

Webster and Kruglanski (1994) had participants who varied in Need for Closure request the number of pages of information they wanted about a job applicant before forming an impression of that person. Although differences in the number of pages requested were found, participants did not receive the information they requested. Instead, all participants viewed a video of the applicant and then formed their impressions.

What would have happened if participants had actually received the information that they requested? Participants high in Need for Closure requested fewer pages of information than did participants low in Need for Closure, so it is possible that the impressions they developed would have differed greatly. In addition, people may develop different impressions as a function of whether they can select the amount of information they receive. Johnston and Macrae (1994), for example, found that when participants were given a set amount of information about a group, information that both confirmed and disconfirmed their stereotypes, those stereotypes were weakened. In contrast, when participants were able to seek out the information that they wanted, they generally sought stereotype-confirming information, and so their stereotypes about the group did not change (see also Trope \& Thompson, 1997).

This led us to perform two studies. In Study 1, participants had an opportunity to learn about as many group members as they wanted before forming a group stereotype. In Study 2, participants were given information about a set number of group members before forming a group stereotype. By manipulating information in this way, we were able to investigate how both individual differences and information can affect stereotype development. 


\section{Study 1}

In Study 1, we investigated the relations among Personal Need for Structure, Need for Closure, and Personal Fear of Invalidity, as well as the effects of these individual differences on stereotype development, when participants could seek as much information as they wanted while developing stereotypes about a group. Three aspects of stereotype development were of interest: information seeking, stereotype content, and stereotype confidence.

The stereotype development phase of the study was described as research on the ability to match groups of people with appropriate jobs. Participants were told that they could read about as many or as few members from these groups as they wanted. Then they would be asked some questions about the group. Finally, they would match the entire group of people (not just the group members they read about, but the entire group to which these individuals belonged) with appropriate jobs. The individual difference measures, which participants believed were part of a separate study investigating personality, were completed either before or after the stereotype development phase of our study.

Information seeking was assessed by counting the number of group member descriptions that participants chose to read. Stereotype content included stereotype detail (the absolute number of characteristics participants ascribed to the group) and stereotype accuracy (the degree to which participants described the group as possessing the characteristics that the various individual group members did in fact possess). Finally, stereotype confidence was how confident participants were that the stereotypes they developed indeed described the group.

We hoped to learn whether (1) individuals who differed in Personal Need for Structure, Need for Closure, or Personal Fear of Invalidity would choose to read about different numbers of group members while developing group stereotypes; (2) the stereotypes these individuals developed would differ in content, particularly in their levels of detail and accuracy; and (3) these individuals would have different levels of confidence in the stereotypes they developed. Based on past research, we expected Need for Closure and Personal Need for Structure to correlate negatively with stereotype accuracy, and Need for Closure to correlate positively with confidence. Because people who are high in Personal Fear of Invalidity are afraid of making incorrect decisions, we also expected Personal Fear of Invalidity to correlate positively with information seeking.

\section{Method}

Participants Seventy-seven University of Western Ontario students (60 women and 17 men) volunteered to participate in two brief and apparently unrelated studies. They received C\$10 for their participation. Participants ranged in age from 17 to 45 years $(M=23.96)$.

\section{Materials}

Individual difference measures We used the revised 11-item version of the Personal Need for Structure Scale (Neuberg, Judice, \& West, 1997; Neuberg \& Newsom, 1993), the 42-item version of the Need for Closure Scale (Webster \& Kruglanski, 1994), and the 14-item version of the Personal Fear of Invalidity Scale (Neuberg, Judice, \& West, 1997). All three measures use a -3 (strongly disagree) to +3 (strongly agree) response format. Responses to the items on each scale were averaged to create overall scores, with higher scores indicating higher levels of need for structure, need for closure, or fear of invalidity. These measures have proven reliable in past research and were reliable in our study as well (Personal Need for Structure $\alpha=.87$; Need for Closure $\alpha=.88$; Personal Fear of Invalidity $\alpha=.82$ ).

Group member descriptions Forty descriptions were created for members of two fictitious groups. Each group contained 20 members: 10 men and 10 women. Each group member description was 9-10 lines long and included the person's name, age, education, characteristic behavior, and current employment. The name, age, education, and current employment information served as filler material to make the person seem more 'real'. More important 
was the middle section of each description: $4-5$ lines containing eight trait adjectives or short phrases that described the group member's personality.

The personality descriptions were derived from the results of a multimethod factor analysis of personality dimensions (the Personality Research Form) and vocational interests (the Strong Vocational Interest Blank). Siess and Jackson (1970) found that these personality dimensions and vocational interests clustered into five factors, each of which included several personality dimensions that were shared by certain groups of professionals. We created two fictitious groups, using the two factors in which the greatest number of jobs and personality dimensions were clustered together. One group was thus based on Siess and Jackson's impulse control factor and the other was based on their human relations management factor.

The impulse control factor consists of such personality dimensions as desire for cognitive structure, order, change (reversed), autonomy (reversed), and impulsivity (reversed), and such vocational interests as accounting and office work. Of these personality dimensions, cognitive structure loaded strongest on this factor (see Siess \& Jackson, 1970). Therefore, all 20 members of one fictitious group were described as being high in cognitive structure (e.g. very precise and rigid). That is, four of the eight trait adjectives or short phrases used to describe each group member were always related to cognitive structure. The other four adjectives or phrases described each person as possessing another personality dimension that loaded on the same factor as cognitive structure, such as (1) order (e.g. organized, methodical), (2) change-reversed (e.g. predictable, consistent), (3) autonomy-reversed (e.g. conforming, dependent), or (4) impulsivityreversed (e.g. calm, careful). Thus, five group members were described as cognitively structured and orderly, five as cognitively structured and not changeable, five as cognitively structured and not autonomous, and five as cognitively structured and not impulsive.

The human relations management factor consists of such personality dimensions as dominance, nurturance, social desirability, exhibition, and affiliation, and such vocational interests as public administration and personnel management. Of these personality dimensions, dominance loaded strongest on this factor (see Siess \& Jackson, 1970). Therefore, all 20 members of the second fictitious group were described as being high in dominance (e.g. strong leaders and controlling). That is, four of the eight trait adjectives or short phrases used to describe each group member were always related to dominance. The other four adjectives or phrases described each person as possessing another personality dimension that loaded on the same factor as dominance, such as (1) nurturance (e.g. sympathetic, comforting), (2) social desirability (e.g. self-presentation, concerned with appearance), (3) exhibition (e.g. entertaining, noticeable), or (4) affiliation (e.g. friendly, sociable). Thus, five group members were described as dominant and nurturing, five as dominant and self-desirable, five as dominant and exhibitionist, and five as dominant and affiliated.

The 40 group member descriptions were pilot tested with an independent sample of 28 participants who were similar to the participants in the actual study. In the pilot study, participants read each description and then rated its favorability and the extent to which it was associated with the personality dimensions of interest. We then selected group member descriptions that (1) were relatively favorable, (2) made the relevant personality traits evident, (3) produced little variance in favorability and trait descriptiveness ratings, and (4) involved no significant sex differences in participants' ratings. Based on the pilot testing, we selected 24 descriptions for use in the main study, 12 descriptions per group (6 male, 6 female).

Stereotype measures We used a closed-ended stereotype measure, containing the adjectives from the group member descriptions, to assess the content of the developed stereotypes. This measure was a list of 20 personality trait adjectives. Ten of these described the impulse control factor (6 traits relating to cognitive structure and 1 trait relating to each of the 
other dimensions) and 10 of them described the human relations management factor (6 traits relating to dominance and 1 trait relating to each of the other dimensions). Participants were asked to rate how well each trait described the group on a 7-point scale, where 1 was 'not at all descriptive' and 7 was 'extremely descriptive'. If participants did not know whether a trait was descriptive or not, they were asked to give that trait a rating of 0 (these traits were not included in the accuracy scores described below). Participants rarely used these zero ratings and the use of such ratings was not systematically related to any of the other variables. To determine stereotype accuracy, we computed mean descriptiveness ratings for the traits that were associated with the relevant personality dimensions.

Esses et al.'s (1993) open-ended stereotype measure was also used to assess stereotype content and confidence. Participants were asked to list the characteristics of each group and then to indicate how confident they were that each characteristic described that group $(0 \%-100 \%)$. Because the closed-ended measure only included trait adjectives, we focused on traits provided in the open-ended measure when looking at stereotype content, so that the two measures would be comparable. ${ }^{1}$ Stereotype detail, therefore, was the number of traits participants ascribed to each group. The more traits participants listed as descriptive of the groups, the more detailed their stereotypes were considered to be. To assess stereotype accuracy, the number of accurate traits was counted. Only traits associated with the relevant personality dimensions were accepted as accurate (opposites were used for the reversescored traits). The proportion of accurate traits was then calculated by dividing this number by the total number of traits that participants ascribed to a group. ${ }^{2}$ Finally, stereotype confidence was calculated as mean confidence ratings for all traits attributed to a group.

Procedure Participants were told that they were participating in two separate studies that would take about 45 minutes in all to complete. Participants were asked to remain for the full 45 minutes in order to deter them from reading only a few group member descriptions just to get out of the experiment early. All participants agreed to remain for the full duration of the study.

The stereotype development phase was explained as a job-matching study investigating how employers try to match different groups of people with suitable jobs. We wanted to create a situation that indirectly required participants to develop group stereotypes without indicating that stereotypes were the focus of our research. The individual difference measures were explained as scales for another professor in the psychology department who needed some normative data. The order of the scales was counterbalanced across participants. The order of the stereotype development and individual differences phases of the study was also counterbalanced across participants.

The job-matching study was performed on a computer. Participants were told that there were two different groups of people that needed to be matched with suitable jobs and that the people in each group belonged together because they had similar demographic characteristics (not personality characteristics), such as family background. We told participants that the computer would take them through the entire procedure for one group (they would match the people in that group with suitable jobs), and then the computer would take them through it again for the second group. The computer randomly determined which group was presented first.

Further instructions were adapted from Johnston and Macrae (1994). Participants were told to read 'as many or as few of the group member descriptions as you need to, in order to get a feel for what the group is like as a whole', and then to 'stop reading the descriptions when you feel that you have enough information to match up the entire group of people with suitable jobs'. When they were done reading about group members, participants expected to answer some questions about the group as a whole and to match the entire group of people with suitable jobs. Participants were not told how many group member descriptions were available in total. 
The study began when the computer displayed a randomly selected group member description. After reading that description, participants chose whether they wanted to read about another group member or go on to answer questions about the group. If participants chose to read about another group member, then another randomly selected description appeared, followed by the same choices about how to proceed. This process continued until participants chose to answer questions about the group. At that point, the computer presented the dependent measures. These were always presented in the same order: the open-ended stereotype measure first, then the closed-ended stereotype measure (followed by a few questions about ideal occupations for the group, in order to maintain the cover story). The procedure was then repeated for the second target group. After both phases of the study (stereotype development and individual difference measures) were completed, participants were probed for suspicion and fully debriefed. No one seemed suspicious about our cover story or could guess the true purpose of the study.

\section{Results}

None of the findings depended on the order of the measures, the order in which groups were presented, the nature of the groups (dominant versus cognitive structure), or the participants' sex or age. Thus, the following analyses did not include these factors.

Relations among the individual differences Personal Fear of Invalidity did not correlate significantly with either Personal Need for Structure or Need for Closure. Personal Need for Structure and Need for Closure, however, were strongly correlated (see Table 1). This finding supports the claims of Neuberg, Judice, and West (1997), and Neuberg, West, Thompson, and Judice (1997), that Personal Need for Structure and Need for Closure may be the same construct. Because the correlation between Personal Need for Structure and Need for Closure $(r(75)=.80, p<.001)$, was nearly as high as the reliability of each measure, the two

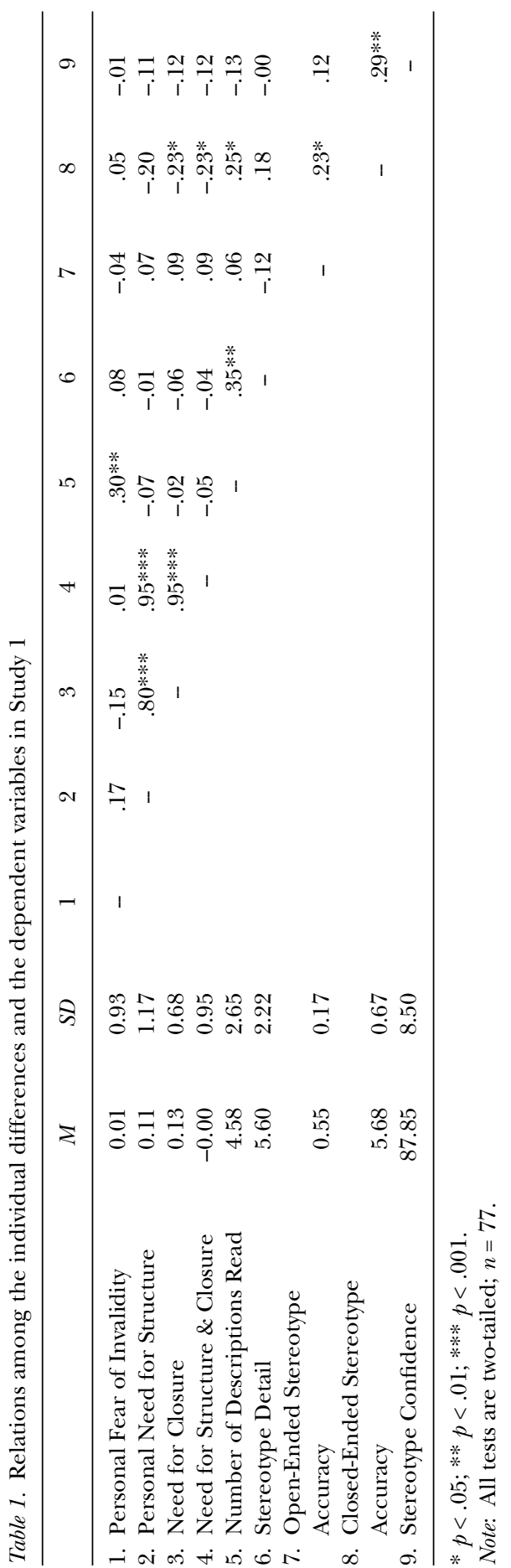


measures were combined by computing $z$-scores for each scale and then averaging them. We labelled this composite variable Need for Structure \& Closure $(\alpha=.92)$.

Information seeking Overall, participants chose to read an average of $4.58(S D=2.65)$ group member descriptions. Personal Fear of Invalidity correlated positively with the number of group member descriptions that participants chose to read $(r(75)=.30, p<.01)$. Based on tertile splits, participants high in Personal Fear of Invalidity read more than 5 group member descriptions $(M=5.31, S D=2.98)$, whereas participants low in Personal Fear of Invalidity read fewer than $4(M=3.87, S D=1.96)$. Need for Structure \& Closure did not correlate significantly with the number of group member descriptions that participants chose to read.

\section{Stereotype content}

Stereotype detail We conducted a regression analysis using Personal Fear of Invalidity, Need for Structure \& Closure, and the number of group member descriptions read to predict stereotype detail. The predictor variables were centered before they were entered into the analysis.

The overall regression analysis was significant $(F(7,69)=2.37, p<.05)$, accounting for $19.36 \%$ of the variance. There was a significant main effect for the number of group member descriptions read $(\beta=.35 ; t(73)=3.06, p<.01)$. Participants who read more group member descriptions provided more detailed stereotypes. There was also a significant interaction between Personal Fear of Invalidity and the number of group member descriptions read $(\beta=.28 ; t(70)=2.02, p<.05)$. To examine the nature of this interaction, predicted stereotype detail was plotted for one standard deviation below and above the mean for Personal Fear of Invalidity and number of group member descriptions read. As shown in Figure 1, participants higher in Personal Fear of Invalidity developed more detailed stereotypes as they read about more group members, whereas participants lower in Personal Fear of Invalidity developed equally detailed stereotypes no

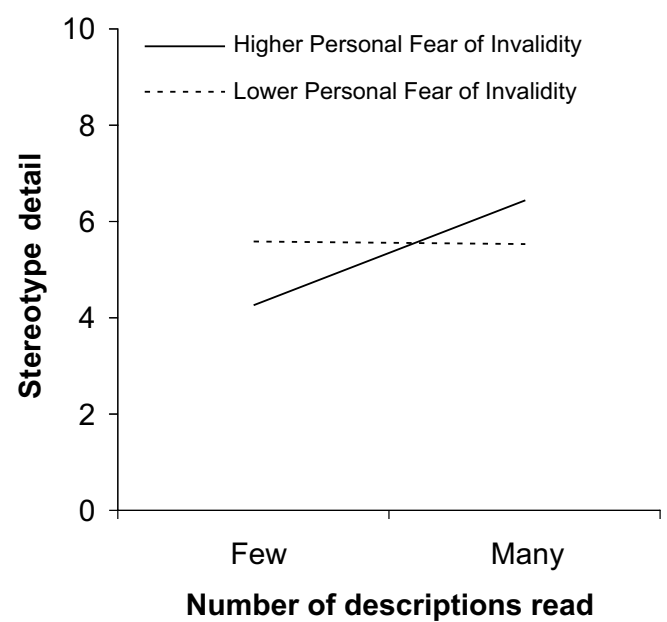

Figure 1. The interaction between Personal Fear of Invalidity and the number of group member descriptions read as predictors of stereotype detail in Study 1.

matter how many descriptions of group members they read.

Stereotype accuracy We also conducted regression analyses using Personal Fear of Invalidity, Need for Structure \& Closure, and the number of group member descriptions read to predict accuracy on the open-ended and closed-ended stereotype measures. The predictor variables were again centered before they were entered into the analyses.

On the open-ended measure, the overall regression analysis was not significant $(F<1)$ and none of the individual effects was significant. The overall regression analysis was not significant for the closed-ended measure either $(F(7,69)=1.54, n s)$, though the model including only the main effects was significant $(F(3$, $73)=2.93, p<.05)$, accounting for $10.76 \%$ of the variance. In particular, there was a significant effect for the number of group member descriptions read $(\beta=.24 ; t(73)=2.10, p<.05)$. The more group members participants read about, the more they rated accurate traits as descriptive of the group. In addition, there was a marginally significant effect for the Need for Structure \& Closure $(\beta=-.21 ; t(73)=-1.92$, 
$p<.06)$. Participants higher in Need for Structure \& Closure tended to rate accurate traits as less descriptive than did participants lower in Need for Structure \& Closure.

Stereotype confidence Finally, we conducted a regression analysis using Personal Fear of Invalidity, Need for Structure \& Closure, and the number of group member descriptions read as predictors of stereotype confidence. The predictor variables were again centered before they were entered into the analyses.

The overall regression analysis was significant $(F(7,69)=2.74, p<.05)$, accounting for $21.76 \%$ of the variance. There was a significant interaction between Need for Structure \& Closure and the number of group member descriptions that were read $(\beta=-.29 ; t(70)=$ $-2.16, p<.05)$. To examine the nature of this interaction, predicted stereotype confidence was plotted for one standard deviation below and above the mean for Need for Structure \& Closure and number of group member descriptions read. As shown in Figure 2, participants higher in Need for Structure \& Closure reported less stereotype confidence when they read about more group members, whereas

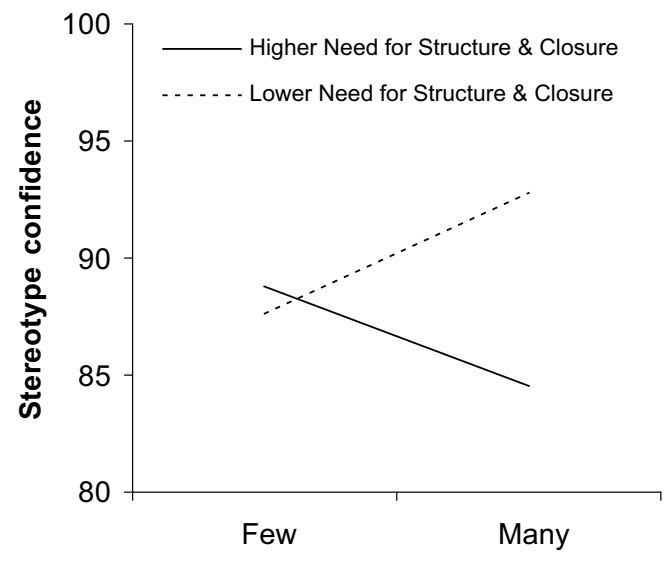

Number of descriptions read

Figure 2. The interaction between Need for Structure $\&$ Closure and the number of group member descriptions read as predictors of stereotype confidence in Study 1. participants lower in Need for Structure \& Closure reported more confidence. There was also a significant interaction between Personal Fear of Invalidity and the number of group member descriptions that were read $(\beta=-.29$; $t(70)=-2.15, p<.05)$. To examine the nature of this interaction, predicted stereotype confidence was also plotted for one standard deviation below and above the mean for Personal Fear of Invalidity and number of group member descriptions read. As shown in Figure 3, participants higher in Personal Fear of Invalidity reported less stereotype confidence when they read about more group members, whereas participants lower in Personal Fear of Invalidity reported more confidence.

\section{Study 2}

In Study 2, we investigated the relations among the individual differences (Personal Need for Structure, Need for Closure, Personal Fear of Invalidity) and stereotype development when participants were given a specific number of group member descriptions to read prior to reporting group stereotypes. We were primarily interested in the effect this would have on

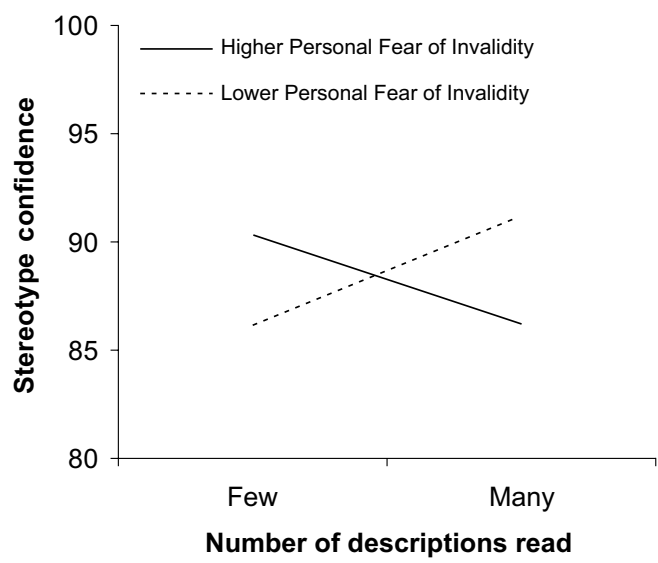

Figure 3. The interaction between Personal Fear of Invalidity and the number of group member descriptions read as predictors of stereotype confidence in Study 1. 
stereotype content and confidence. The cover story and materials from Study 1 were used again. The main methodological difference was that in Study 1, participants could read about as many group members as they wanted, whereas participants in Study 2 were randomly assigned to an information condition where they would read about two, four, or eight group members.

The results of Study 1 suggested that two group member descriptions are fewer than most people would choose to read, whereas eight descriptions are more than most people would choose to read. Therefore, we presented people with two or eight group member descriptions in order to examine the effects of minimal and maximal information on stereotype development. In addition, Personal Fear of Invalidity was the only individual difference that correlated with information seeking in Study 1. On average, participants low in Personal Fear of Invalidity chose to read less than four descriptions and participants high in Personal Fear of Invalidity chose to read more than four descriptions. We thus included four group member descriptions as an information condition as well.

In Study 2, we wanted to learn whether (1) the number of group member descriptions given to participants would influence the content and confidence of their stereotypes; (2) participants who differed in Personal Need for Structure, Need for Closure, or Personal Fear of Invalidity would develop stereotypes that differed in content and confidence; and (3) these individual differences would interact with the number of group member descriptions participants received to influence stereotype content and confidence. Based on the results from Study 1, we expected that the number of group member descriptions read would correlate positively with stereotype detail and accuracy, and perhaps would interact with Personal Fear of Invalidity, Need for Closure, and Personal Need for Structure to affect stereotype detail and accuracy. Based on previous research, we also expected Need for Closure and Personal Need for Structure to correlate negatively with stereotype accuracy, and Need for Closure to correlate positively with stereotype confidence.

\section{Method}

Participants 184 introductory psychology students (117 women and 67 men) from the University of Western Ontario volunteered to participate in two brief and apparently unrelated studies for course credit. Participants ranged in age from 17 to 28 years $(M=19.29)$.

Procedure The cover study and materials were all the same as those used in Study 1. The individual difference scales were somewhat less reliable than previous reports (Personal Need for Structure $\alpha=.67$; Need for Closure $\alpha=.76$; Personal Fear of Invalidity $\alpha=.81$ ), though this problem was remedied when Personal Need for Structure and Need for Closure were combined (see below). Stereotype content (including stereotype detail and stereotype accuracy) and stereotype confidence were assessed as in Study 1. Table 3 presents the means and standard deviations for these variables in each information condition.

There was only one major procedural change in Study 2-participants were randomly assigned (by the computer) to read two, four, or eight descriptions of group members. As in Study 1, participants were probed for suspicion and fully debriefed. No one seemed suspicious about our cover story or could guess the true purpose of the study.

\section{Results}

None of the findings depended on the order of the measures, the order in which groups were presented, the nature of the groups (dominant versus cognitive), or the participants' sex or age. Thus, the following analyses did not include these factors.

Relations among the individual differences Personal Fear of Invalidity was correlated positively with Personal Need for Structure $(r(183)$ $=.25, p<.001)$, but did not correlate significantly with Need for Closure. Need for Closure and Personal Need for Structure were again correlated very strongly $(r(183)=.74, p<.001)$ (see Table 2). Because the correlation between Need for Closure and Personal Need for Structure was so high, we again combined these two 
measures $(\alpha=.84)$. Personal Fear of Invalidity did not correlate significantly with the resulting Need for Structure \& Closure variable.

\section{Stereotype content}

Stereotype detail We conducted a regression analysis using Personal Fear of Invalidity, Need for Structure \& Closure, and the number of group member descriptions read to predict stereotype detail. The individual difference variables were centered before they were entered into the analyses.

The overall regression analysis was marginally significant $(F(7,177)=1.79, p<.09)$, accounting for $6.61 \%$ of the variance. There was a significant main effect for the number of group member descriptions read $(\beta=.16$; $t(181)=2.14, p<.05)$. The more descriptions participants read, the more detailed were the stereotypes that they developed. There was also a significant interaction between Personal Fear of Invalidity and the number of group member descriptions read $(\beta=-.20 ; t(178)=-2.67$, $p<.01)$. To examine the nature of this interaction, predicted stereotype detail was plotted for one standard deviation below and above the mean for Personal Fear of Invalidity at each level of the number of group member descriptions read. As shown in Figure 4, participants higher in Personal Fear of Invalidity reported equally detailed stereotypes no matter how many group member descriptions they read, whereas participants lower in Personal Fear of Invalidity reported more detailed stereotypes when they read more group member descriptions.

Stereotype accuracy We conducted regression analyses using Personal Fear of Invalidity, Need for Structure \& Closure, and the number of group member descriptions read to predict stereotype accuracy on the open-ended and closed-ended measures. The individual difference variables were again centered before they were entered into the analyses.

On the open-ended measure, the overall regression analysis was significant $(F(7,177)=$ $3.96, p<.001)$, accounting for $13.53 \%$ of the variance. There was a significant main effect for

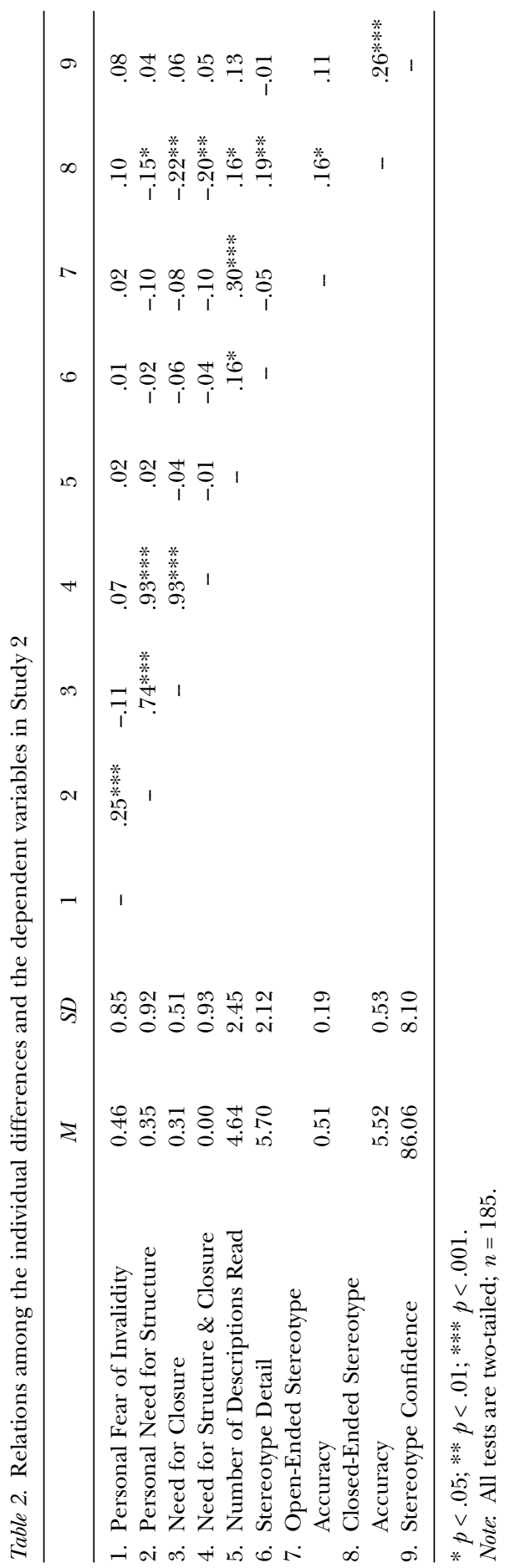


Table 3. Means for the dependent variables as a function of number of descriptions read in Study 2

\begin{tabular}{|c|c|c|c|}
\hline & \multicolumn{3}{|c|}{ Number of descriptions read } \\
\hline & 2 & 4 & 8 \\
\hline Stereotype Detail & $5.23(1.74)$ & $5.75(2.39)$ & $6.10(2.08)$ \\
\hline Open-Ended Stereotype Accuracy & $0.44(0.20)$ & $0.51(0.15)$ & $0.58(0.18)$ \\
\hline Closed-Ended Stereotype Accuracy & $5.41(0.51)$ & $5.51(0.53)$ & $5.63(0.52)$ \\
\hline Stereotype Confidence & $84.67(10.21)$ & $86.13(7.49)$ & $87.37(6.05)$ \\
\hline
\end{tabular}

Note: Numbers in parentheses indicate standard deviations.

the number of group member descriptions $\operatorname{read}(\beta=.30 ; t(181)=4.25, p<.001)$. Participants who read more group member descriptions listed a greater proportion of accurate traits. There was also a significant interaction among Personal Fear of Invalidity, Need for Structure \& Closure, and the number of group member descriptions read $(F(7,177)=-2.10$, $p<.05)$. To examine the nature of this interaction, predicted open-ended stereotype accuracy was plotted for one standard deviation below and above the mean for Personal Fear of Invalidity and Need for Structure \& Closure at each level of the number of group member descriptions read. The results are shown in Figure 5. When two group member descriptions were read, participants higher in Personal Fear of Invalidity and lower in Need for Structure \&

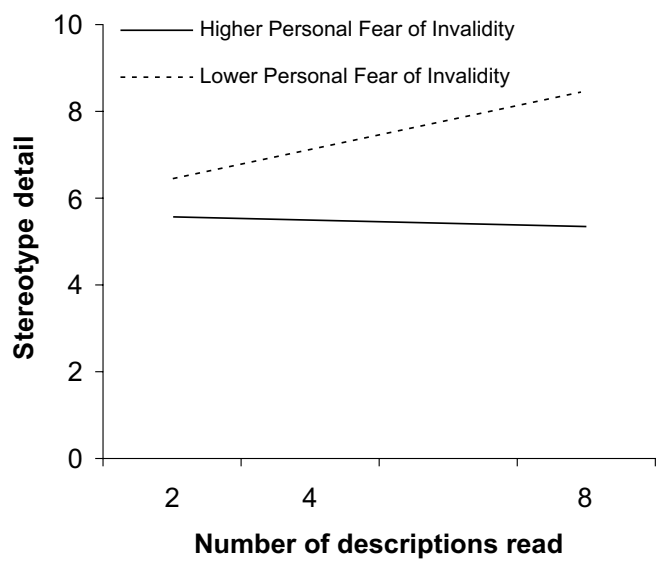

Figure 4. The interaction between Personal Fear of Invalidity and the number of group member descriptions read as predictors of stereotype detail in Study 2.
Closure generated the most accurate stereotypes, whereas participants higher in both Personal Fear of Invalidity and Need for Structure \& Closure generated the least accurate stereotypes. When four group member descriptions were read, participants did not seem to differ in the accuracy of the stereotypes that they generated as a function of their Personal Fear of Invalidity and Need for Structure \& Closure. Finally, when eight group member descriptions were read, participants higher in Personal Fear of Invalidity and lower in Need for Structure \& Closure again seemed to generate the most accurate stereotypes, whereas participants lower in Personal Fear of Invalidity generated the least accurate stereotypes-regardless of their level of Need for Structure \& Closure.

The overall regression analysis of the closedended measure was significant $(F(7,177)=$ $2.84, p<.01$ ), accounting for $10.09 \%$ of the variance. There was a significant main effect for the number of group member descriptions $\operatorname{read}(\beta=.16 ; t(181)=2.23, p<.05)$. The more group member descriptions participants read, the more they rated accurate traits as descriptive of the group. There was also a significant main effect for the Need for Structure \& Closure $(\beta=-.20 ; t(181)=-2.84, p<.01)$. Participants higher in Need for Structure \& Closure rated the accurate traits as less descriptive of the groups than did participants lower in Need for Structure \& Closure.

Stereotype confidence We also conducted a regression analysis using Personal Fear of Invalidity, Need for Structure \& Closure, and the number of group member descriptions 

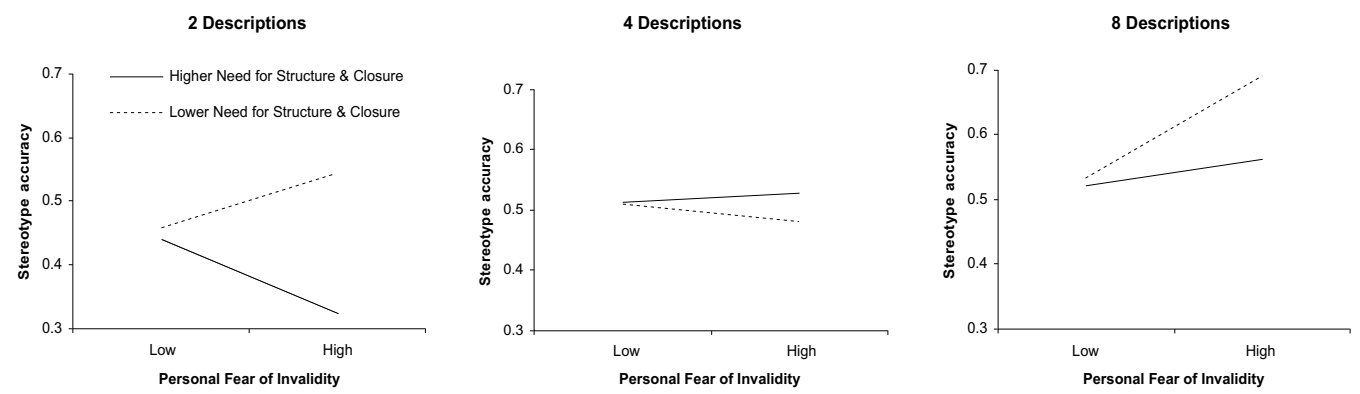

Figure 5. The interaction between Personal Fear of Invalidity and Need for Structure \& Closure as predictors of open-ended stereotype accuracy at 2, 4, and 8 group member descriptions read in Study 2.

read as predictors of stereotype confidence. Again, the individual difference variables were centered before they were entered into the analysis.

The overall regression analysis was not significant $(F(7,177)=1.52)$, accounting for just $5.67 \%$ of the variance. An examination of the individual effects revealed, however, a significant interaction between Need for Structure \& Closure and number of group member descriptions read $(\beta=-.16 ; t(178)=-2.08, p<.05)$. To examine the nature of this interaction, predicted stereotype confidence was plotted for one standard deviation below and above the mean for Need for Structure \& Closure and the number of group member descriptions read. As shown in Figure 6, participants higher in Need for Structure \& Closure did not differ in stereotype confidence as a function of how many group member descriptions they read, whereas participants lower in Need for Structure \& Closure were more confident when they read more group member descriptions.

\section{General discussion}

As in previous research (Neuberg, Judice, \& West, 1997; Neuberg, West, Judice, \& Thompson, 1997), Personal Need for Structure and Need for Closure were strongly related in both studies, suggesting that they may be indistinguishable. The findings from these two studies go beyond previous research, however, by demonstrating that Personal Fear of Invalidity, Personal Need for Structure, and Need for
Closure can affect stereotype development. One unique feature of our research is that we allowed participants to gather the amount of information they desired about group members in one study, and manipulated the amount of information they received in another. Across studies, we found that participants developed more detailed and more accurate stereotypes when they received more information. Clearly, giving people more information about group members affects the content of the stereotypes that they develop and makes group perception more accurate-whether that amount of information is what people desire or not.

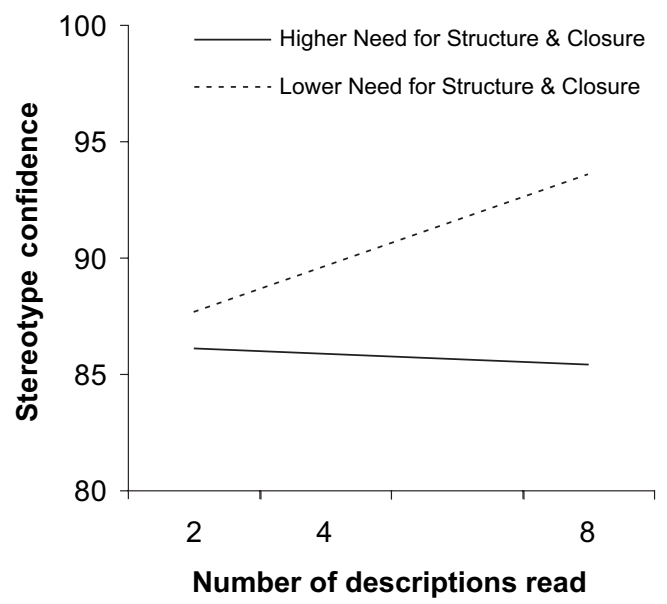

Figure 6. The interaction between Need for Structure $\&$ Closure and the number of group member descriptions read as predictors of stereotype confidence in Study 2. 
Significant relations were also found between the individual differences and several components of stereotype development. Stereotype detail, stereotype accuracy, and stereotype confidence were all affected. Thus, the degree to which people are afraid of making mistakes (Personal Fear of Invalidity), want to keep their lives simple and structured (Personal Need for Structure), and seek answers without ambiguity or confusion (Need for Closure) can indeed affect how group stereotypes develop.

The effects of Personal Fear of Invalidity differed somewhat across studies. In Study 1, participants higher in Personal Fear of Invalidity chose to read about more group members, but the stereotypes they developed were no more accurate than those developed by their peers. In addition, participants higher in Personal Fear of Invalidity were less confident about their stereotypes when they read more descriptions, whereas participants lower in Personal Fear of Invalidity were more confident. In Study 2, participants with different levels of Personal Fear of Invalidity were equally accurate and confident in the stereotypes they developed, whether they were given two, four, or eight descriptions of group members to read. Thus, although participants higher in Personal Fear of Invalidity wanted more information than did their peers in Study 1, the results from Study 2 suggest that they did not need this extra information to develop comparably accurate and confident stereotypes.

Personal Fear of Invalidity also affected stereotype detail, and its effects differed across studies as well. In Study 1, participants higher in Personal Fear of Invalidity developed more detailed stereotypes when they received more information, whereas participants lower in Personal Fear of Invalidity developed equally detailed stereotypes regardless of how much information they received. In Study 2, however, participants lower in Personal Fear of Invalidity developed more detailed stereotypes when they received more information, whereas participants higher in Personal Fear of Invalidity developed equally detailed stereotypes, regardless of how much information they received. The only difference between the studies was that participants in Study 1 chose the amount of information they received, whereas participants in Study 2 were randomly assigned to receive different amounts of information. It appears that having control over the information one receives is key. If participants can choose how much information they receive, those higher in Personal Fear of Invalidity choose to receive more information, and develop more detailed stereotypes when they receive more information. The amount of information received doesn't seem to affect those lower in Personal Fear of Invalidity under these circumstances. If participants cannot choose how much information they receive, however, those higher in Personal Fear of Invalidity do not develop more detailed stereotypes when they receive more information, whereas those lower in Personal Fear of Invalidity do.

Need for Structure \& Closure had somewhat different effects. In general, it was negatively related to stereotype accuracy, a finding that replicates past research (e.g. Moskowitz, 1993; Schaller et al., 1995; Webster \& Kruglanski, 1994). Across studies, participants higher in Need for Structure \& Closure rated accurate traits as less descriptive of the groups on the closed-ended measure than did their peers. A more complex pattern, involving an interaction among Need for Structure \& Closure, Personal Fear of Invalidity, and the number of descriptions read, was found on the open-ended accuracy measure, but only in Study 2. It seemed that when participants were given two descriptions (which was probably less information than they wanted) or eight descriptions (which was probably more information than they wanted), those who were low in Need for Structure \& Closure and high in Personal Fear of Invalidity generated the most accurate stereotypes. This suggests that people who do not need to keep their lives simple and structured, but are afraid of making mistakes, may be especially likely to develop accurate stereotypes when they are provided with amounts of information that seem insufficient or excessive.

Need for Structure \& Closure also interacted with the number of group member descriptions read to affect stereotype confidence. Across 
studies, participants lower in Need for Structure \& Closure were more confident about their stereotypes when they had more information about group members, whereas amount of information did not affect or reduced the confidence of participants higher in Need for Structure \& Closure. Apparently, receiving more information can boost the confidence of people who are lower in Need for Structure \& Closure only.

\section{Implications}

Our findings indicate that individual differences in information processing can affect stereotype development. Differences in how people develop stereotypes may also lead to differences in stereotype usage and/or change. For example, how confident people are about the stereotypes they develop may influence how likely they are to base decisions on stereotype information and whether they will change their stereotypes in the face of new information. Thus, understanding differences in how people develop group stereotypes may lead to important insights into when different people will rely on their stereotypes, or how to persuade different people to change their stereotypes.

Our results also speak to whether researchers should give participants control over the information they receive in the laboratory. In our daily activities, we can sometimes gather as much information as we want before making decisions about groups. If this is the sort of 'real world' scenario that researchers are trying to investigate in the laboratory, then participants should be able to seek out as much information as they want-rather than being presented with a set amount of information. But if researchers are trying to assess stereotype development when people have only limited information, then they should control the amount of information that participants receive. As the somewhat different results from Study 1 and Study 2 suggest, it is important to distinguish between these two types of situations. The content of the stereotypes that develop might differ depending upon whether participants can control the amount of information they receive.
The results from our studies also have implications for such issues as media portrayals of immigrant or minority groups (or any groups to which people have limited exposure). Study 1 suggests that if people are able and motivated to learn about a group, then they will generally seek out enough information to develop accurate stereotypes. Some people and communities experience little cultural diversity, however, so they may not have as much information as they would like. Others may get their information from the news, where it comes as a set 'package' that sometimes contains more or less information than they want.

Study 2 suggests that when people are presented with little information, they are less likely to develop accurate stereotypes, yet they develop stereotypes anyway and are confident about them. If the information they receive is biased, then such effects could be devastatingpeople develop highly inaccurate stereotypes, but are confident about them, so they are unlikely to correct them. Televised news has the potential to be especially damaging in this way, for broadcasts are often brief and intentionally devised to be engaging and eye-catching.

\section{Limitations and future research}

A better test of the effects of allowing participants to control the amount of information that they receive about group members would be to manipulate that control within the same experiment, rather than allowing participants control in one study and no control in another. In such a study, the results from participants who can control the amount of information they receive could be compared directly with the results from participants who lack that control.

In addition, information in our studies was operationalized as written descriptions of individual group members. Findings might differ in a study where participants develop stereotypes based on characteristics that are inferred from the actual behavior of individual group members. In such a study, other processes (e.g. behavioral confirmation) might occur in parallel with information processing. 


\section{Notes}

1. For this reason, demographic information was not included in the trait accuracy index for the open-ended measure.

2. The findings did not differ when we analyzed stereotype accuracy using trait totals, means, or percentages.

\section{Acknowledgments}

The research presented in this article was supported by a research grant from the Social Sciences and Humanities Research Council of Canada to Victoria Esses. We would like to thank Jim Olson, Sam Paunonen, Richard Moreland, and three anonymous reviewers for their helpful comments on an earlier version of this article.

\section{References}

Chaiken, S., Liberman, A., \& Eagly, A. H. (1989). Heuristic and systematic information processing within and beyond the persuasion context. In J. S. Uleman \& J. A. Bargh (Eds.), Unintended thought (pp. 212-252). New York: Guilford.

Dijksterhuis, A., \& van Knippenberg, A. (1999). On the parameters of associative strength: Central tendency and variability as determinants of stereotype accessibility. Personality and Social Psychology Bulletin, 25, 527-536.

Esses, V. M., Haddock, G., \& Zanna, M. P. (1993). Values, stereotypes, and emotions as determinants of intergroup attitudes. In D. M. Mackie \& D. L. Hamilton (Eds.), Affect, cognition, and stereotyping: Interactive processes in group perception (pp. 137-166). San Diego, CA: Academic Press.

Fiske, S. T., \& Neuberg, S. L. (1990). A continuum of impression formation, from category-based to individuating processes: Influences of information and motivation on attention and interpretation. In M. P. Zanna (Ed.), Advances in experimental social psychology (Vol. 23, pp. 1-74). New York: Academic Press.

Hamilton, D. L., \& Trolier, T. K. (1986). Stereotypes and stereotyping: An overview of the cognitive approach. In J. F. Dovidio \& S. L. Gaertner (Eds.), Prejudice, discrimination, and racism (pp. 127-163). San Diego, CA: Academic Press.

Johnston, L. C., \& Macrae, C. N. (1994). Changing social stereotypes: The case of the information seeker. European Journal of Social Psychology, 24, 581-592.

Kruglanski, A. W. (1989). Lay epistemics and human knowledge: Cognitive and motivational bases. New York: Plenum.

Kruglanski, A. W., Atash, M. N., DeGrada, E., Mannetti, L., Webster, D. M., \& Pierro, A. (1997). Psychological theory testing versus psychometric nay-saying: Comment on Neuberg et al.'s (1997) critique of the need for closure scale. Journal of Personality and Social Psychology, 73, 1005-1016.

Macrae, C. N., Milne, A. B., \& Bodenhausen, G. V. (1994). Stereotypes as energy-saving devices: A peek inside the cognitive toolbox. Journal of Personality and Social Psychology, 66, 37-47.

McConnell, A. R., Sherman, S. J., \& Hamilton, D. L. (1997). Target entitativity: Implications for information processing about individual and group targets. Journal of Personality and Social Psychology, 72, 750-762.

Meiser, T., \& Hewstone, M. (2001). Crossed categorization effects on the formation of illusory correlations. European Journal of Social Psychology, 31, 443-466.

Moskowitz, G. B. (1993). Individual differences in social categorization: The influence of personal need for structure on spontaneous trait inferences. Journal of Personality and Social Psychology, 65, 132-142.

Neuberg, S. L., Judice, T. N., \& West, S. G. (1997). What the need for closure scale measures and what it does not: Toward differentiating among related epistemic motives. Journal of Personality and Social Psychology, 72, 1396-1412.

Neuberg, S. L., \& Newsom, J. T. (1993). Personal need for structure: Individual differences in the desire for simple structure. Journal of Personality and Social Psychology, 65, 113-131.

Neuberg, S. L., West, S. G., Judice, T. N., \& Thompson, M. M. (1997). On dimensionality, discriminant validity, and the role of psychometric analyses in personality theory and measurement: Reply to Kruglanski et al.'s (1997) defense of the need for closure scale. Journal of Personality and Social Psychology, 73, 1017-1029.

Ryan, C. S., \& Bogart, L. M. (1997). Development of new group members' in-group and out-group stereotypes: Changes in perceived variability and ethnocentrism. Journal of Personality and Social Psychology, 73, 719-732.

Ryan, C. S., Bogart, L. M., \& Vender, J. P. (2000). Effects of perceived group variability on the gathering of information about individual group members. Journal of Experimental Social Psychology, 36, 90-101.

Schaller, M., Boyd, C., Yohannes, H., \& O’Brien, M. (1995). The prejudiced personality revisited: 
Personal need for structure and formation of erroneous group stereotypes. Journal of Personality and Social Psychology, 68, 544-555.

Sherman, J. W. (2001). The dynamic relationship between stereotype efficiency and mental representation. In G. B. Moskowitz (Ed.), Cognitive social psychology: The Princeton symposium on the legacy and future of social cognition (pp. 177-190). Mahwah, NJ: Erlbaum.

Siess, T. F., \& Jackson, D. N. (1970). Vocational interests and personality: An empirical integration. Journal of Counseling Psychology, 17, 27-35.

Stroessner, S. J., \& Plaks, J. E. (2001). Illusory correlation and stereotype formation: Tracing the arc of research over a quarter century. In G. B. Moskowitz (Ed.), Cognitive social psychology: The Princeton symposium on the legacy and future of social cognition (pp. 247-259). Mahwah, NJ: Erlbaum.

Thompson, M. M., Naccarato, M. E., \& Parker, K. E. (1989, May). Assessing cognitive need: The development of the Personal Need for Structure and Personal Fear of Invalidity Scales. Paper presented at the annual meeting of the Canadian Psychological Association, Halifax, Nova Scotia, Canada.

Trope, Y., \& Thompson, E. P. (1997). Looking for the truth in all the wrong places? Asymmetric search of individuating information about stereotyped group members. Journal of Personality and Social Psychology, 73, 229-241.

Webster, D. M., \& Kruglanski, A. W. (1994). Individual differences in need for cognitive closure. Journal of Personality and Social Psychology, 67, 1049-1062.

Paper received 24 August 2000; revised version accepted 30 April 2005.

\section{Biographical note}

KIMBERLEY A. CLOW is an assistant professor in psychology at the University of Ontario Institute of Technology. Her primary research interest is in social categorization and stereotyping and the majority of her work is influenced by a socialcognitive perspective.

VICTORIA M. ESSES is a professor of psychology at the University of Western Ontario. Her primary research focus is on intergroup relations, such as stereotyping, discrimination, and prejudice. She is particularly interested in issues related to immigrants and refugees. 